\title{
Traumatic stress and psychopathology: experiences of a trauma clinic
}

\author{
U Subramaney \\ Division of Psychiatry, Department of Neurosciences, University of the Witwatersrand, and Centre for the Study of Violence and \\ Reconciliation (CSVR), Johannesburg, South Africa
}

\begin{abstract}
Objective: The study was undertaken to investigate the profile of individuals referred to the psychiatrist for assessment in terms of their demographic features, trauma experienced, diagnosis according to the fourth edition of the Diagnostic and Statistical Manual of Mental Disorders (DSM IV), and recommendations made by the psychiatrist. Method: The trauma clinic, a key component of the Victim Empowerment Programme (VEP) of the Centre for the Study of Violence and Reconciliation (CSVR) in Johannesburg, South Africa is a multidisciplinary unit offering counselling and debriefing services to victims/survivors of violence. The staff consists of trained therapists (clinical, research and educational psychologists), social workers and a psychiatric nurse. Since 1998, a psychiatrist has been consulting at the clinic as well. A retrospective case review of all intake notes and progress notes of the clients was undertaken. The period of the survey was 1999-2002. Results: Of 3668 individuals presenting to the centre, 127 were referred to the resident psychiatrist for assessment. 119 were assessed. The majority were in the age range 15-45, unmarried and unemployed. Children under the age of 15 and refugees constituted 9.9\% and 30\% respectively. Major Depression was the most commonly diagnosed psychiatric condition (17.6\%), with Post Traumatic Stress Disorder (PTSD), diagnosed less than expected (5.9\%). Co- morbidity was common. Medication was prescribed for $66.4 \%$ of the sample, with a further 5.9\% hospitalised. Conclusion: Not all individuals exposed to trauma develop PTSD, with mood disorders possibly being more common.
\end{abstract}

Keywords: Traumatic stress; Post traumatic stress disorder; refugees; complex trauma; Major Depression; xenophobia

Received: 16.11 .04

Accepted: 13.07 .05

\section{Introduction}

The trauma clinic, now known as the Victim Empowerment Programme (VEP) within the Centre for the Study of Violence and Reconciliation (CSVR, www.cSvr.org.za), is a multidisciplinary unit offering counselling and debriefing services to victims/survivors of violence. It was set up during the late 1980's as a support service for political detainees during the height of the political struggle in South Africa. At that time, services involved counselling and the provision of a supportive network for detainees who had been subjected to acts of political violence. As the clinic developed, it became increasingly clear that the need for psychological services had outstripped the need for mere social support, and the organisation grew into an internationally recognised non-governmental organisation with a mandate of understanding the causes of and risk factors for political and criminal violence. The organisation became increasingly viewed

\section{Correspondence:}

Dr U Subramaney, Division of Psychiatry,

University of the Witwatersrand, 7 York Road, Parktown, 2193,

Johannesburg, South Africa

email: ugashs@absamail.co.za
}

as a legitimate resource for all victims of crime and violence. Since the democratic elections of 1994, refugees and asylum seekers, particularly from other African countries, also began seeking assistance at the trauma clinic. Since 1998, the multidisciplinary team, consisting of trained therapists (clinical, research and educational psychologists; social workers and a psychiatric nurse) expanded to include a psychiatrist. The reason for this was that the therapists were seeing an increasing number of individuals who had complicated diagnostic issues, as well as individuals who were not responding to trauma counselling.

This study was undertaken to examine the demographic and clinical profile of individuals who presented to the trauma clinic, in particular, those who were referred for psychiatric assessment.

\section{Method}

A retrospective record review of the intake and progress notes of all individuals seen at the trauma clinic and referred to the psychiatrist was undertaken. The period the survey was 1999 to 2002. Data were analysed according to demographic characteristics, types of trauma experienced, clinical diagnosis according to DSM IV and outcome in terms of recommendations made by the psychiatrist. The traumatic events were grouped according to Friedman's categorisation of traumatic exposure and experience ${ }^{2}$ as follows: 
1) Catastrophic events involving actual or threatened harm to the self or serious injury. (category I)

2) Those who witnessed the aftermath of a catastrophic event but were never in personal danger. (category II)

3) Those who were confronted with the facts of a life-threatening event. (category III)

A residual category "Other" was used to denote those clientele who did not fit into any of the aforementioned categories, but who presented to the trauma clinic with symptoms warranting psychiatric opinion. Individuals were further distinguished into those who experienced a single traumatic event versus those who were exposed to more than one event or had experienced trauma which was ongoing, e.g. having to look after a spouse who was dying of a terminal illness.

\section{Statistical methodology}

In the analysis of this predominantly descriptive study, use was made of frequencies, percentages, frequency distributions, bar charts, odds ratio (OR) and the 95\% confidence interval for the latter.

\section{Results}

\section{Number of referrals}

A total of 3668 individuals were seen over the four years, with an average of 917 cases per year. The highest number was in 2001 ( $\mathrm{n}=1023)$ and the lowest in 2002 ( $\mathrm{n}=842)$. Of 127 individuals referred, 119 (3.3\%) were ultimately assessed by the psychiatrist. 8 individuals did not attend the assessments, and were lost to follow up.

\section{Frequency distributions of Age and Gender}

Of the referrals assessed, 42\% (50/1 19) were male, $58 \%$ (69/1 19) were female. Most were in the 15-45 year age group, (82\%, 97/ 119), while 10\% (12/1 19) were under the age of 15 and $8 \%$ (10/ 119) were over 45 . The mean age of the sample was 30.2 years (sd $=10.72$ )

\section{Marital Status and Employment}

The majority, $71.4 \%(85 / 119)$ were unmarried, whilst only $28.6 \%$ (34/119) were married.

Unemployment was common -55.5\% (66/1 19). Of those who were employed, $44.5 \%$ (53/1 19), worked mainly in the informal sector as hawkers or street vendors.

\section{Racial distribution and geographical origin}

The majority of the sample, 70\% ( $\mathrm{n}=83$ ) were of South African origin, with $41 \%(n=49)$ being black, $14 \%(n=17)$ white, $8 \%(n=9)$ asian and $7 \%(\mathrm{n}=8)$ coloured. Non South Africans constituted the remaining $30 \%$ of the sample. All but two of these (one British, one Chinese) were refugees from other African countries.

The numbers of refugees who were seen at the trauma clinic increased from 5\% ( $\mathrm{n}=1)$ of the total sample in 1999 to 34.2 $\%(n=13)$ in the year 2000, and to 53.1\% $(n=17)$ in 2001. In 2002 this dropped to $13.7 \%(n=4)$.

\section{Trauma Exposure}

A category I event was experienced by $61.4 \%(n=73)$ of the sample, a category II event by 5\% ( $\mathrm{n}=6)$ and a category III event by $26.9 \%(n=32) .6 .7 \%(n=8)$ represented the residual category. In the sample, $57.1 \%(n=68)$ of individuals had experienced a single traumatic event, while $42.9 \%(n=51)$ had experienced more than one event. In this population, individuals who experienced more than one traumatic event were 1.93 times (odds ratio) more likely to have developed PTSD than those who experienced a single event. The 95\% confidence interval for the OR is (0.81 - 4.63)

\section{Diagnosis}

Major Depression (MDD) was diagnosed in 17.6\% (n=21) of the sample, while only 5.9\% ( $n=7)$ satisfied criteria for PTSD. Acute Stress Disorder was diagnosed in 2.5\% ( $\mathrm{n}=3$ ) of the sample. 51 clients (42.9\%) had diagnoses other than those mentioned i.e.: $\mathrm{v}$ codes ( $n=30,25.2 \%)$, adjustment disorders ( $n=18,15.1 \%)$ and psychotic disorders $(n=3,2.5 \%)$. The high rate of other diagnoses suggests that the experience of a traumatic event is often associated with disorders other than PTSD or Major Depression.

More than one diagnosis was made in 37 cases (31.1\%). These include Axis I and Axis II co-morbidity, as well as different Axis I disorders. A dual diagnosis of MDD +PTSD was made in $20.2 \%$ ( $n=24$ ) of the sample. Other dual diagnoses included PTSD and borderline personality disorder $(0.8 \%, n=1)$ and MDD together with personality and substance use disorders (10.1\%, $\mathrm{n}=12$ ). There is a suggestion that the presence of one disorder may lead to increased susceptibility to another psychiatric diagnosis.

\begin{tabular}{|l|l|l|l|l|}
\hline \multicolumn{6}{|l|}{ Table I: Number of clients seen over the four years } \\
\hline Year & Total cases & Cases Referred & Assessed & \% Assessed \\
\hline 1999 & 880 & 20 & 20 & 2.3 \\
2000 & 923 & 40 & 38 & 4.1 \\
2001 & 1023 & 32 & 32 & 3.2 \\
2002 & 842 & 35 & 29 & 3.4 \\
\hline Total & 3668 & 127 & 119 & 3.3 \\
\hline
\end{tabular}

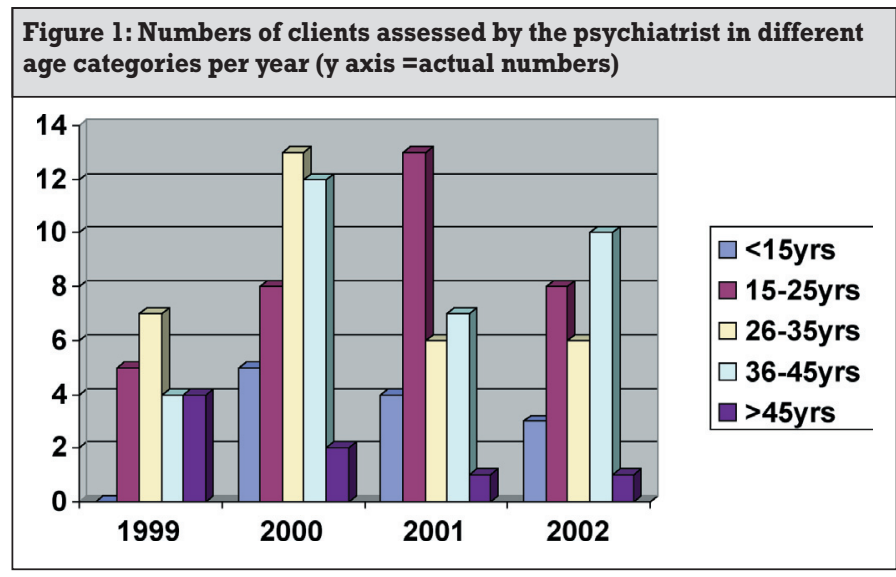

Figure 2: Numbers of clients per category of trauma exposure (1999-2002)

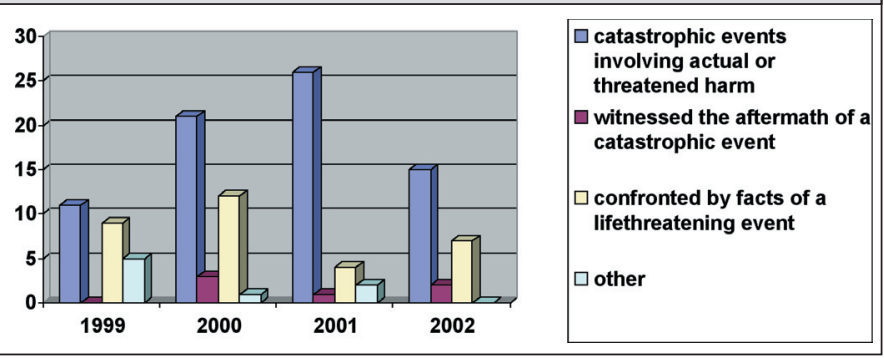




\section{Treatment recommendations}

$66.4 \%(n=79)$ were referred to community mental health services for medication, with an antidepressant (selective serotonin reuptake inhibitor) recommended in all cases. A further $5.9 \%(n=7)$ of the sample was referred to hospital for admission. Hence 72.3\% ( $n=86)$ of those assessed by the psychiatrist warranted intervention beyond counselling. $25.2 \%$, $(n=30)$ required trauma counselling. Other resources e.g. Narcotics and Alcoholics Anonymous were recommended for the remainder of the sample $(2.5 \%, n=3)$.

\section{Discussion}

The lifetime risk of exposure to trauma in general populations is as high as 90\%. ${ }^{3}$ Not all exposed however, will develop PTSD. ${ }^{3,4}$ In their Detroit Area Survey of Trauma, Breslau et al examined trauma and subsequent risk for PTSD (as defined in DSM IV) in a community sample of 2181 persons aged $18-45$ years. ${ }^{4}$ The lifetime prevalence of exposure to one or more traumatic events was $89.6 \%$. Key aetiological questions in PTSD research concerns the subsequent risk, or "conditional risk", of developing PTSD.

The conditional risk of PTSD is defined as "the probability of developing PTSD in persons exposed to trauma". " Estimates of the conditional risk of PTSD are derived from information on the prevalence of exposure and the proportion of those exposed who meet criteria for PTSD. This conditional risk has a broad range (9$65 \%$ ) depending on factors related to the person, and characteristics related to the trauma itself. ${ }^{3,4}$

In the United States, PTSD has been shown to have a lifetime prevalence of $8 \%$, while there is a much higher prevalence in countries affected by civil war, genocide, forced migration and terrorism. ${ }^{3}$ In this study, only 3.5\% of the total population who attended the trauma clinic were referred to the psychiatrist for an assessment. The vast majority was seemingly not thought to be ill enough to warrant a referral. It would be interesting to know whether any of these had psychopathology which was missed, i.e. should have been referred and were not; or whether in fact the vast majority of those who experience any form of trauma do not warrant psychiatric assessment. Of those who were assessed, the finding that only 5.9\% received a diagnosis of PTSD is in keeping with findings in the literature. ${ }^{3,4,5}$ In a study by Kessler et al, the rate of PTSD appeared to be influenced in part by the nature of the event.3 Rape was the trauma most often associated with the development of PTSD(approximately $50 \%$ of people developed PTSD following rape, regardless of gender, whereas the rate of PTSD following a natural disaster was 5\%). This study did not specifically analyse the relationship between specific events and PTSD, however it did demonstrate that the number of traumatic events play a role, with multiple traumatic events being associated with a diagnosis of PTSD more often than a single episode of trauma. This has been shown in previous studies. ${ }^{4}$ In the current study, Major Depression was more commonly diagnosed than PTSD. This may be due to a variety of factors, including the nature of the trauma experienced, the nature of the diagnostic criteria used, and underreporting of symptoms. However, comorbid diagnoses (PTSD and MDD) were made in $20.2 \%$ of the 119 individuals assessed. High rates of comorbidity between Major Depression and PTSD have also been found in numerous previous studies. ${ }^{3,4,6}$ This raises a question as to whether PTSD and depression are independent consequences of trauma exposure, with separate pathways leading to distinct psychiatric disorders. The finding of other dual diagnoses ( MDD with Personality and substance use disorders; PTSD and borderline personality disorder) suggests that in certain patients, the presence of one disorder may lead to increased susceptibility to another psychiatric diagnosis. Unfortunately, it is difficult to speculate as to whether there was anything unique or different about those patients who had dual diagnoses compared with those who had a single diagnosis. The current study also found a high rate of other diagnoses (v codes, adjustment disorders and psychotic disorders). This may suggest that the experience of a traumatic event does not necessarily lead to a diagnosis of PTSD or MDD, but may act as a precipitant for other psychiatric conditions.

The transformation process in South Africa has been associated with an influx of refugees and asylum seekers from other African countries. Unpublished research by the author has shown that the refugee community in South Africa is particularly vulnerable to trauma due to experiences in their home countries, as well as experiences related to xenophobia in their host country. The provision of social networks and support structures are crucial protective factors which assist the individual in regaining his/her functioning after trauma. ${ }^{8}$ These are sadly lacking within refugee communities.

The limitations of research of this nature must be borne in mind. Firstly, this was a retrospective chart review involving cross-sectional analysis i.e. single assessment. One psychiatrist assessed all the individuals, therefore reliability of data is dependent upon that clinician's judgement. Ideally, a prospectively designed study where assessments are made by more than one researcher using rating scales as well as clinical assessments should be used.

\section{Conclusion}

The data obtained in this study supports the notion that not all individuals exposed to trauma will develop PTSD, and that other diagnoses, notably major depression may be more prominent. More than one traumatic event compared to a single event was more likely to be associated with a diagnosis of PTSD. Those assessed psychiatrically tended to have severe psychopathology, with more than a third of the sample recommended to receive medication. Considering that only $3 \%$ of the clients seen at the clinic were referred for psychiatric assessment, the likelihood of psychopathology having been missed by counsellors cannot be excluded and suggests that, perhaps even in these settings, more thorough screening and diagnostic procedures are required.

\section{References}

1. American Psychiatric Association. Diagnostic and Statistical Manual of Mental Disorders. 4th Edition. Washington DC: American Psychiatric Association; 1994.

2. Friedman MJ. Post Traumatic Stress Disorder. Kansas City, MO:Compact Clinicals, 2001.

3. Kessler R, Sonnega A, Bromet E, Hughes M, Nelson CB. Post Traumatic Stress Disorder in the National Comorbidity Survey. Arch Gen Psychiatry 1995; 52:1048-1060.

4. Breslau N. The Epidemiology of Post Traumatic Stress Disorder: what is the extent of the problem? J Clin Psychiatry 2001; 62(suppl 17) : 16-22.

5. Friedman MJ. Pharmacological management of Post Traumatic Stress Disorder. Primary Psychiatry 2003; 10(8): 66-73.

6. Davidson JRT, Hughes D, Blazer DG, George LK. Post-traumatic Stress Disorder in the community: an epidemiological study. Psych Med 1991; 21:713-721.

7. Subramaney, U, Spencer F. Refugees and Psychopathology. Presentation at the World Association of Social Psychiatry Congress. March 2004.

8. Kleber RJ, Figley CR and Gersons BPR. Beyond Trauma: Cultural and Societal Dynamics. The International Society for Traumatic Stress Studies. New York: Plenum Press, 1995. 